\title{
Solving Fundamental Solution of Non-Homogeneous Heat Equation with Dirichlet Boundary Conditions
}

\author{
Kahsay Godifey Wubneh \\ Department of Mathematics, Wollo University, Amhara, Ethiopia \\ kahsayg29@gmail.com
}

\begin{abstract}
Keywords: Heat equation, Non-homogeneous Heat Equation, gamma function and Louville equation.
\end{abstract}

\begin{abstract}
In this study, we developed a solution of nonhomogeneous heat equation with Dirichlet boundary conditions. moreover, the non-homogeneous heat equation with constant coefficient. since heat equation has a simple form, we would like to start from the heat equation to find the exact solution of the partial differential equation with constant coefficient. to emphasize our main results, we also consider some important way of solving of partial differential equation specially solving heat equation with Dirichlet boundary conditions. the main results of our paper are quite general in nature and yield some interesting solution of non-homogeneous heat equation with Dirichlet boundary conditions and it is used for problems of mathematical modeling and mathematical physics.
\end{abstract}

\section{Introduction}

Differential equations are used to construct more models of reality and these modeling suggests that some solutions of the differential equations with variable coefficients using different methods There are more methods of solving different researchers applied to solve heat equation by different method in this paper we find a solution of nonhomogeneous heat equation with Dirichlet Boundary conditions.

Many physical problems such as wave equation, heat equation, Poisson equation and Laplace equation are modeled by differential equations which are an example of partial differential equations. some partial differential equations have numerical solution and exact solution in regular shape domain but in this paper, we will try to solve exact solution of non-homogeneous heat equation. Then in this paper we try to solve the exact solution of nonhomogeneous heat equation with Dirichlet Boundary conditions.

Heat equation is a superposition principle of solutions and therefore from a stock of simple solutions it is possible to build solutions to complex problems. Thus, the temperature distribution in a body can be considered to be due to the additive influence of the various internal,external and boundary agents affecting the heat flow. There are in fact special solutions to the heat equation which are sufficiently fundamental that solutions to very broad categories of heat conduction problems can be written immediately in terms of these fundamental solutions to the differential equation.

We recall, an equation containing the derivatives or differentials of one or more dependent variables in relation to one or more independent variables is called a partial differential equation (abbreviated to PDE).

Note that the order of a partial differential equation is the degree of the highest order derivatives in the equation. For instance, if there are two independent variables $(x, y)$, a partial differential equation of second order has the general form[1].

A differential equation of the form

$$
\frac{\partial u}{\partial t}=k^{2} \frac{\partial^{2} u}{\partial x^{2}}+f(x, t)
$$

Equation (1.1) is known as parabolic linear second order partial differential equation heat equation with one dimension 
The initial condition will be of the form

$$
u(x, 0)=f(x, t), a<x<b
$$

And the boundary Dirichlet Boundary conditions will be in the form

$$
u(a, t)=C_{1}, u(b, t)=C_{2}, 0 \leq x \leq d
$$

When we solving a partial differential equation, we will need initial or boundary value problems to get the particular solution of the partial differential equation.

\section{Preliminaries}

The non-homogeneous heat equation arises when studying heat equation problems with a heat source we can now solve this equation. The ideas in the proof are very important to know about the solution of non-homogeneous heat equation.

Theorem1.The solution of the inhomogeneous heat equation

$$
u_{t}(x, t)=\Delta u+f(x, t),(t>0,-\infty<x<\infty)
$$

With initial condition

$$
u(x, 0)=0,(-\infty<x<\infty)
$$

Is given by

$$
u(x, t)=\int_{0}^{t} \int_{-\infty}^{\infty} \phi(x-y, t-s) f(y, s) d y d s
$$

where

$$
\phi(x, t)=\frac{1}{2 \sqrt{\pi t}} e^{-X^{2} /\left(4 C^{2} t\right)} \text { if } t>0 \text { and }-\infty<x<\infty
$$

Remark 1: The inner integral in Eqn (2.3) is the convolution of the kernel with $f(x, s)$, where $S$ is fixed. The process of expressing the solution of the non-homogeneous heat equation Eqn (2.1) as an integral (in $t)$ of the solution of the homogeneous heat equation with initial condition. $f(x, s),(s>$ 0 fixed ) is called Duhamel'sprinciple.

PROOF: It is clear that we can immediately simplifies the situation to the case of zero initial data

$$
u_{t}=\Delta u+f
$$

With initial conditions $\quad(t>0,-\infty<x \infty) ; u(0)=0$

It turns out that the solution to this problem can be obtained by solving a family of homogenous problems. We illustrate the idea in the situation of ODE systems first. Consider the ODE

$$
v^{\prime}-\Delta v=f, \quad \text { and } \quad v(0)=0
$$

In this case we can multiply both sides of the equation (2.6) by $e^{-A t}$ gives

$$
\frac{d}{d t}\left(e^{-A t} v\right)=e^{-A t} f(t), \text { and }\left(e^{-A t} V\right)(0)=0
$$

Integrating both sides of equation (1.7) we have 


$$
\begin{gathered}
\int\left[\frac{d}{d t}\left(e^{-A t} v\right)\right] d t=\int\left[e^{-A t} f(t)\right] d t \\
V(t)=e^{A t} \int_{0}^{t} e^{-A t} f(s) d s=\int_{0}^{t} e^{A(t-s)} f(s) d s
\end{gathered}
$$

Now consider the homogeneous system;

$$
V^{\prime}-A V=0, \quad V(0)=g
$$

One easily see that

$$
\mathrm{V}(\mathrm{t})=\mathrm{e}^{\mathrm{At}} \mathrm{g}
$$

Comparing the two results we see that the solution to the non-homogeneous equation with zero initial value can be represented as a summation of the solution of the solution of a family of homogenous equation with nonzero initial value

$$
V(t)=\int_{0}^{t} W(t ; s) d s
$$

With $W(t ; s)$ satisfies the homogenous equation (2.10) with initial time $s$ an initial value $f(s)$. This is the Duhamel's principle.

By this principle we can write down the solution

$$
\begin{aligned}
U(x, t) & =\int_{0}^{t} \int_{-\infty}^{\infty} \phi(x-y, t-s) f(y, s) d y d s \\
& =\int_{0}^{t} \frac{1}{4 \pi(t-s)^{n / 2}} \int_{-\infty}^{\infty} e^{-\frac{(x-y)^{2}}{4(t-s)}} f(y, s) d y d s
\end{aligned}
$$

Theorem 2:( solution of non-homogenous heat equation) Let $f \in C_{1}^{2}(t>0,-\infty<x<\infty)$ and have compact support then
a) $U \in C_{1}^{2}\left(\mathbb{R}^{n} \times(0, \infty)\right)$
b) $-\Delta U=f$ for $t>0$
c) for each $x_{0} \in \mathbb{R}^{n}, \lim _{(x, t) \rightarrow\left(X_{0}, 0\right)} U(x, t)=0,\left(x \in \mathbb{R}^{n}, t>0\right.$

Proof

a) From eq (1.12) we have

$$
U(x, t)=\int_{0}^{t} \int_{-\infty}^{\infty} \phi(y, s) f(x-y, t-s) d y d s
$$

Assumption we can differentiate inside the integrals;

$$
\begin{gathered}
U_{t}(x, t)=\int_{-\infty}^{\infty} \phi(y, s) f(x-y, 0) d y+\int_{0}^{t} \int_{-\infty}^{\infty} \phi(y, s) f_{t}(x-y, t-t) d y d s \\
\partial_{x_{i} x_{j}} U(x, t)=\int_{0}^{t} \int_{-\infty}^{\infty} \phi(y, s)\left(\partial_{x_{i} x_{j}} f\right)(x-y, t-s) d y d s
\end{gathered}
$$

We calculate

$$
U_{t}(x, t)-\Delta U(x, t)=\int_{0}^{t} \int_{-\infty}^{\infty} \phi(y, s)\left[\left(\partial_{t}-\Delta\right) f(x-y, t-s)\right] d y d s
$$




$$
\begin{aligned}
& +\int_{-\infty}^{\infty} \phi(y, s) f(x-y, 0) d y d s \\
& \quad=A+B
\end{aligned}
$$

where $A=\int_{0}^{t} \int_{-\infty}^{\infty} \phi(y, s)\left[\left(\partial_{t}-\Delta\right) f(x-y, t-s)\right] d y d s \quad$ and

$$
B=\int_{-\infty}^{\infty} \phi(y, s) f(x-y, 0) d y d s
$$

b) First note that since [2]

$$
\left|\int_{-\infty}^{\infty} \phi(y, s)\left[\left(\partial_{t}-\Delta\right) f(x-y, t-s)\right] d y\right| \leq \sup \left(\left|\left(\partial_{t}-\Delta\right) f\right|\right) \int_{-\infty}^{\infty} \phi(y, s) d y
$$

The integral is well defined, as $\phi$ is singularity at $S=0$ we write

$$
A=\int_{0}^{t} \int_{-\infty}^{\infty} \phi(y, s)\left[\left(\partial_{t}-\Delta\right) f(x-y, t-s)\right] d y d s
$$

(by using improper integral as $\varepsilon \rightarrow 0$ the lower boundary of the integral starts from $\varepsilon$ )

$$
\begin{gathered}
=\lim _{\varepsilon \rightarrow 0}\left[\int_{\varepsilon}^{t} \int_{-\infty}^{\infty} \phi(y, s)\left[\left(-\partial_{s}-\Delta_{y}\right) f(x-y, t-s)\right] d y d s\right] \\
=\int_{-\infty}^{\infty}\left[\left(\partial_{s}-\Delta_{y}\right)\right] \phi(y, s) f(x-y, t-s) d y d s+\int_{-\infty}^{\infty} \phi(y, \varepsilon) d y-\int_{-\infty}^{\infty} \phi(y, t) f(x-y, 0) d y \\
=\lim _{\varepsilon \rightarrow 0} \int_{-\infty}^{\infty} \phi(y, \varepsilon) f(x-y, t-\varepsilon) d y-B
\end{gathered}
$$

Therfore

$$
\begin{gathered}
U_{t}-\Delta U=A+B \\
=\lim _{\varepsilon \rightarrow 0} \int_{-\infty}^{\infty} \phi(y, \varepsilon) f(x-y, t) d y+\int_{-\infty}^{\infty} \phi(y, \varepsilon)[f(x-y, t-\varepsilon) f(x-y, t)] d y=f(x, t)
\end{gathered}
$$

c) It is clear that[2]

$$
\sup _{x}|u(x, t)| \leq t_{(x, t)}^{\sup _{1}}|f| \rightarrow 0 \text {, as } t \rightarrow 0
$$

Combining the above results, we can present the formula for the solution in the general case in the whole space;

The required solution is

$$
U(x, t)=\int_{-\infty}^{\infty} \phi(x-y, t) g(y) d y+\int_{0}^{t} \int_{-\infty}^{\infty} \phi(x-y, t-s) f(y, s) d y d s
$$

As an application of the Theorem above, we consider the effect of a heat source localized at one point on the bar. The solution will be expressed in terms of the special function known as the incomplete gamma function and defined by

$$
\tau(x, t)=\int_{x}^{\infty} e^{-t} t^{a-1} d t \quad(x \geq 0, a>0)
$$

If $x=0$, then $\tau(0, t)=\tau(a)$ where $\tau(a)$ is the gamma function evaluated at $a$.if $x>0$, part of the integral defining $\tau(a)$ is missing, which explains the name of the function. Hence the integral of the 
incomplete gamma function is positive, the values of the integral increases the size of the interval of integration. Thus for all $x \geq 0$,

$$
0<\tau(a, x) \leq \int_{0}^{\infty} e^{-t} t^{a-1} d t=\tau(a)
$$

The integral of the incomplete gamma function converges for all if $x>0$.If $a \geq 0$, we integrate by part

$$
\left(u=e^{-t}, d v=t^{a-1}, d u=-e^{-t}, v=\frac{t^{a}}{a}\right)
$$

And we get

$$
\int_{x}^{\infty} e^{-t} t^{a-1} d t=\frac{1}{a} e^{-t} t^{a}+\frac{1}{a} \int_{x}^{\infty} e^{-t} t^{a} d t=\frac{e^{-t} x^{a}}{a}+\frac{1}{a} \int_{x}^{\infty} e^{-t} t^{a} d t
$$

In particular, if $-1<a<0$ and $x>0$, we can write

$$
\int_{x}^{\infty} e^{-t} t^{a-1} d t=\frac{1}{a}\left[-e^{-t} x^{a}+\tau(a+1, x)\right] \text {, where } \tau(a+1, x)=\int_{x}^{\infty} e^{-t} t^{a} d t
$$

Remark 2: boundary value problem

$$
U_{t}=U_{x x}+\delta_{0}(x)
$$

With boundary conditions $(t>0,-\infty<x<\infty), U(x, 0)=0(-\infty<x<\infty)$ In equation (1), $\delta_{0}(x)$ represented a source of heat that is applied at the point $x, \forall t>0$

Solution: Applying the above theorem with $f(x, t)=\delta_{0}(x)$ we find

$$
U(x, t)=\frac{1}{\sqrt{\pi}} \int_{0}^{t} \int_{-\infty}^{\infty} \frac{1}{\sqrt{t-s}} e^{-(x-y)^{2} /(t-s)} \delta_{0}(y) d y d s
$$

Integrating against $\delta_{0}(y)$ in the integral yields

$$
U(x, t)=\frac{1}{\sqrt{\pi}} \int_{0}^{t} \frac{1}{\sqrt{\pi}} e^{-x^{2} /(t-s)} d s
$$

To simplify the exponential function we make a change of variables:

Putting $z=\frac{x^{2}}{t-s}$ and differentiating both sides we get

$$
d z=\frac{d}{d s}\left(\frac{x^{2}}{t-s}\right)=x^{2} \frac{d}{d s}\left(\frac{1}{t-s}\right)=x^{2} \frac{d}{d s}(t-s)^{-1}=-x^{2}(-1)(t-s)^{-2}=\frac{x^{2}}{(t-s)^{2}} d s
$$

Or it can be write by rearranging as $d s=\frac{x^{2}}{z^{2}} d z$, and $\frac{1}{\sqrt{t-s}}=\frac{\sqrt{z}}{|x|}$

As $s$ varies from 0 to $t$ and $z$ varies from $\frac{x^{2}}{t}$ to $\infty$ so

$$
U(x, t)=\frac{|x|}{\sqrt{\pi}} \int_{\frac{x^{2}}{t}}^{\infty} e^{-z} z^{-3 / 2} d z
$$

Applying the incomplete gamma function with $a=-\frac{1}{2}$ and $x$ replaced by $\frac{x^{2}}{t}$, we find that $x \neq 0$ 


$$
u(x, t)=2 \frac{\sqrt{t}}{\sqrt{x}} e^{-x^{2} / t}-2 \frac{|x|}{\sqrt{\pi}} \tau\left(\frac{1}{2}, \frac{x^{2}}{t}\right)
$$

Since $\mathrm{u}$ is calculated (4) by integrating a positive function, it is positive for all $(x, t)$.moreover, since $2 \frac{|x|}{\sqrt{\pi}} \tau\left(\frac{1}{2}, \frac{x^{2}}{t}\right) \geq 0$, it follows that for $t>0$ and all $x$ :

$$
0<u(x, t) \leq 2 \frac{|x|}{\sqrt{\pi}} e^{-x^{2} / t}
$$

3. Fundamental Solution of One-Dimensional Heat Equation with Dirichlet Boundary Conditions We consider a general, non-homogeneous, parabolic initial boundary value problem with nonhomogeneous boundary conditions. The hyperbolic problem is treated in the same way. Let $U$ be a solution of the problem

$$
\left\{\begin{array}{cc}
r(x) m(t) u_{t}-\left[(p(x) u)_{x}+q(x) u\right]=F(x, t), & a<x<b, t>0 \\
B_{a}(u)=\alpha u(a, t)+\beta u(a, t)=a(t) & t \geq 0 \\
B_{b}(u)=\gamma u(b, t)+\delta u_{x}(b, t)=b(t) & t \geq 0 \\
u(x, 0)=f(x) & 0 \leq x \leq b
\end{array}\right.
$$

We assume that $u$ has the following from

$$
u(x, t)=v(x, t)+w(x, t)
$$

Where $w$ is a smooth function satisfying only the boundary conditions

$$
B_{a}(u)=a(t) \text { and } B_{b}(u)=b(t)
$$

Then $v(x, t)=u(x, t)-w(x, t)$ is a solution of the following problem

$$
\left\{\begin{array}{c}
r(x) m(t) u_{t}-\left[(p(x) u)_{x}+q(x) u\right]=F(x, t), a<x<b, t>0 \\
B_{a}(u)=a(t) \quad t \geq 0 \\
B_{b}(u)=b(t) \quad t \geq 0 \\
u(x, o)=\check{f}(x) \quad a \leq x \leq b
\end{array}\right.
$$

Where

$$
\begin{gathered}
\breve{F}(x, t)=F(x, t)-r(x) w_{t}+\left[\left(p(x) w_{x}\right)\right] \\
\check{f}(x)=f(x)-w(x, 0)
\end{gathered}
$$

Consequently, $v$ is a solution of the, non-homogeneous, parabolic initial boundary value problem with homogeneous boundary conditions to which one can applies the methods from the previous section. In general, a function $w$ the form,

$$
w(x, t)=\left(A_{1}+B_{1} x+C_{1} x^{2}\right) a(t)+\left(A_{2}+B_{2} x+C_{2} x^{2}\right) b(t)
$$

The form of the function $w$ for given boundary conditions $w(x, t)=a(t)+\frac{x}{L} b(t)-a(t)$ where $u(a, t)=a(t)$ and $u(b, t)=b(t)$ is called Dirichlet boundary conditions 
Remark 3: Consider the problem

$$
\left\{\begin{array}{cc}
u_{t}-u_{x x}=e^{-t} \sin 3 x & 0<x<\pi, t>0 \\
u(0, t)=u(\pi, t) & t \geq 0 \\
u(x, 0)=\phi(x) & 0<x<\pi \\
u(x, t)=v(x, t)+w(x, t) &
\end{array}\right.
$$

Since we are dealing with Dirchilet boundary conditions then we take for $(x, t)=\frac{x}{\pi}$. Then the function $v$ has to be the solution of the following problem

$$
\left\{\begin{array}{cc}
v_{t}-v_{x x}=e^{-t} \sin 3 x & 0<x<\pi, t>0 \\
v(0, t)=v(\pi, t) & t \geq 0 \\
u(x, 0)=\phi(x)-\frac{x}{\pi} & 0 \leq x \leq \pi
\end{array}\right.
$$

The eigenvalues and the corresponding eigenfunctions for the associated Sturm- Louville problem.

$$
\left\{\begin{array}{c}
x^{\prime \prime}+\lambda x=0,0<x<\pi \\
x(0)=0 \\
x(\pi)=0
\end{array}\right.
$$

Are given by

$$
\lambda_{n}=n^{2} \text { and } x_{n}(x)=\sin (n x), n \geq 1
$$

Hence, the formal solution $v$ of the above problem is given by the formal Fourier series

$$
v(x, t)=\sum_{n=1}^{\infty} T_{n}(t) \sin (n x)
$$

Differentiating $v$ (formally) with respect to $t$ and twice with respect to $x$, and substituting into the equation we get

$$
v_{t}-v_{x x}=\sum_{n=1}^{\infty}\left[T_{n}^{\prime}(t)+n^{2} T_{n}(t)\right] \sin (n x)=e^{-t} \sin (3 x)
$$

This implies that

$$
T^{\prime}(t)+n^{2} T_{n}(t)= \begin{cases}0 & n \neq 3, n \geq 1 \\ e^{-t} & n=3\end{cases}
$$

Moreover at, $\mathrm{t}=0$

$$
\phi(x)-\frac{x}{\pi}=v(x, 0)=\sum_{n=1}^{\infty} T_{n}(0) \sin (n x)
$$

So that

$$
T_{n}^{\prime}(0)=\frac{2}{\pi} \int_{0}^{\pi}\left[\phi(x)-\frac{x}{\pi}\right] \sin (n x)
$$

If $n \neq 3$

$$
T_{n}(t)=T_{n}(0) e^{-n^{2} t}
$$

If $n=3$, then we look for the solution $T_{n}(t)$ of the form

$$
T_{n}(t)=A(t) e^{-9 t}
$$


Then

$$
\frac{d T_{3}(t)}{d t} T_{3}^{\prime}(t)+9 \frac{d T_{3}(t)}{d t}=\frac{d A(t)}{d t} e^{-9 t}-9 A(t) e^{-9 t}+9 A(t) e^{-9 t}=\frac{d A(t)}{d t} e^{-9 t}=e^{-t}
$$

So that

$$
\frac{d A(t)}{d t}=e^{8 t}
$$

And hence integrating both sides we get

$$
A(t)=A(0)+\int_{0}^{t} e^{8 s} d s=A(0)+\frac{1}{8}\left[e^{8 t}-1\right]
$$

Hence

$$
\frac{d T_{3}(t)}{d t}=A(0) e^{-9 t}+\frac{1}{8}\left[e^{8 t}-1\right] e^{-9 t}
$$

Moreover $T_{3}(0)=A(0)$

Hence

$$
v(x, t)=\sum_{n=1}^{\infty} T_{n}(t) \sin (n x)=\frac{1}{8}\left[e^{8 t}-1\right] e^{-9 t}+\sum_{n=1}^{\infty} T_{n}(0) \sin (n x)
$$

And

$$
\begin{aligned}
u(x, t)= & \frac{x}{\pi}+\frac{1}{8}\left[e^{8 t}-1\right] e^{-9 t}+\sum_{n=1}^{\infty} T_{n}(0) e^{-n^{2} t} \sin (n x) \\
& =\frac{x}{\pi}+\frac{1}{8}\left[e^{-t}-e^{-9 t}\right] \sum_{n=1}^{\infty} T_{n}(0) e^{-n^{2} t} \sin (n x)
\end{aligned}
$$

\section{Conclusion}

This paper thus, consisted of a brief overview of what a solution of heat equation is, and what is it used for based on the work has been done on the paper, it can be constructed the solution of nonhomogeneous heat equation with Dirichlet boundary conditions.

A solution of non-homogeneous heat equation was constructed using superposition principles through combining simple solutions of the no-homogenous heat equation. Therefore, the solution of non-homogeneous heat equation derived and to derive this we have used different concepts as theorem and remarks of non-homogeneous equations.

However, in this study we considered a solution for non-homogeneous heat equation with Dirichlet boundary conditions and as we see it simple and easy to derive. Moreover, this procedure can be possibly applied for homogeneous heat equations with Dirichlet boundary conditions. 


\section{References}

[1] Kahsay G., Teklay H., Derivation of Fundamental Solution of Heat Equation by using Symmetry Reduction, IJITEE., Volume-9 Issue-5 (2020), 2278-3075.

[2] L. C. Evans : Partial differential equations (2006)

[3] W. A. Strauss : Partial differential equations (1992)

[4] Erwin Kreyzing, Advanced engineering mathematics, 10th ed., Wiley, 2000.

[5] Alan Jeffrey, Advanced Engineering Mathematics, Academic press, 2002.

[6] Dennis G. Zill and Micheal R. Cullen, Advanced engineering mathematics, 2nd ed. 2000, Jones and Bartlett publisher.

[7] Murray R. Spiegel, Schaum's Outline Series theory and Problems of Advanced Mathematics for Engineers \& Scientists, SI (metric) edition, 1971.

[8] Martin Braun, Differential equations and their applications, Springer-Verlag, 1993.

[9] Morris Tenenbaum and Harry pollard, ordinary differential equation, Dover, 1963.

[10] Dennis G. Zill, A first course in differential equation with modeling application, Ninth ed

[11] N. Pothanna and P. Aparna, "UNSTEADY THERMOVISCOUS FLOW IN A POROUS SLAB OVER AN OSCILLATING FLAT PLATE

", Journal of porous media, vol.22(5), pp.531-543, 2019.

[12] N. Pothanna , P. Aparna, and R.S.R. Gorla, "A NUMERICAL STUDY OF COUPLED NONLINEARNON-LINEAR EQUATIONS OF THERMO-VISCOUS FLUID FLOW IN CYLINDRICAL GEOMETRY",Int. J. of Applied Mechanics and Engineering, vol.22, No.4, pp.965-979,2017.

[13] N. Pothanna and P. Aparna, J C T A, 9(19) 2016, pp. 9049-9054 "UnsteadyUnsteady Forced Oscilations of a FluidBounded by Rigid Bottom", International Journal of Control Theory and Applications, vol.9(19) , pp. 9049-9054, 2016. 